\title{
1 Multivariate Volatility Models
}

\author{
Matthias R. Fengler and Helmut Herwartz
}

\author{
Institute for Statistics and Econometrics, \\ Department of Business Administration and Economics, \\ Humboldt-Universität zu Berlin, \\ Spandauer Straße 1, 10187 Berlin, Deutschland
}

Multivariate Volatility Models belong to the class of nonlinear models for financial data. Here we want to focus on multivariate GARCH models. These models assume that the variance of the innovation distribution follows a time dependent process conditional on information which is generated by the history of the process. In this chapter we demonstrate how to use the bigarch quantlet of XploRe to estimate the conditional covariance of a bivariate (high frequency) return process. In particular we consider a system of exchange rates of two currencies measured against the US Dollar (USD), namely the Deutsche Mark (DEM) and the British Pound Sterling (GBP). For this example process we compare the dynamic properties of the bivariate model with univariate GARCH specifications where cross sectional dependencies are ignored. Moreover, to illustrate the scope of the bivariate model we employ the estimated model to price call options written on foreign exchange rates.

We gratefully acknowledge financial support from the Deutsche Forschungsgemeinschaft, Sonderforschungsbereich 373 "Quantifikation und Simulation ökonomischer Prozesse". 


\subsection{Introduction}

Volatility clustering, i.e. positive correlation of price variations observed on speculative markets, motivated the introduction of autoregressive conditionally heteroskedastic $(\mathrm{ARCH})$ processes by Engle (1982) and its popular generalizations by Bollerslev (1986) (Generalized ARCH, GARCH) and Nelson (1991) (exponential GARCH, EGARCH). A further stylized feature of empirical price variations is contemporaneous cross correlation among a set of assets, stock market indices, exchange rates etc.

Cross section relationships are often implied by economic theory. Interest rate parities, for instance, provide a close relation between domestic and foreign bond rates. Long- and short term interest rates are also related. Assuming absence of arbitrage opportunities the so-called triangular equation formalizes the equality of an exchange rate between two currencies on the one hand and an implied rate constructed via exchange rates measured towards a third currency. Furthermore, as illustrated in Hafner and Herwartz (1998), stock prices of firms acting on the same market often show similar patterns in the sequel of news that are important for the entire market. Similarly, analyzing global volatility transmission Engle, Ito and Lin (1990) and Hamao, Masulis and Ng (1990) found evidence in favor of volatility spillovers between the world's major trading areas occurring in the sequel of floor trading hours. From this point of view, when modeling time varying volatilities, a multivariate model appears to be a natural framework to take cross sectional information into account. Moreover, the covariance between financial assets is of essential importance in finance. Effectively, many problems in financial practice like portfolio optimization, hedging strategies, or Value-at-Risk evaluation require multivariate volatility measures, see e.g. Bollerslev et al. (1988) and Cecchetti, Cumby and Figlewski (1988).

\subsubsection{Model specifications}

Let $\varepsilon_{t}=\left(\varepsilon_{1 t}, \varepsilon_{2 t}, \ldots, \varepsilon_{N t}\right)^{T}$ denote an $N$-dimensional error process. For generalizing the univariate GARCH model towards a multivariate framework, it is necessary to condition the covariance matrix of $\varepsilon_{t}$ on the information set generated by the history of this process. For convenience we regard $\varepsilon_{t}$ as observable error terms or as estimated residuals of a multivariate regression model. The 
process $\varepsilon_{t}$ follows a multivariate GARCH process if it has the representation

$$
\varepsilon_{t}=\Sigma_{t}^{1 / 2} \xi_{t}
$$

where $\Sigma_{t}$ is measurable with respect to information generated up to time $t-1$ $\left(\Omega_{t-1}\right)$, and the $N$ components of $\xi_{t}$ follow a multivariate Gaussian distribution with mean zero and covariance matrix equal to the identity matrix.

The conditional covariance matrix, $\Sigma_{t}=\mathrm{E}\left[\varepsilon_{t} \varepsilon_{t}^{T} \mid \Omega_{t-1}\right]$, has typical elements $\sigma_{i j}$ with $\sigma_{i i}, i=1, \ldots, N$, denoting conditional variances and off-diagonal elements $\sigma_{i j}, i, j=1, \ldots, N, i \neq j$, denoting conditional covariances. To make the specification in (1.1) feasible a parametric description relating $\Sigma_{t}$ to $\Omega_{t-1}$ is necessary. In a multivariate setting, however, dependencies of the second order moments in $\Sigma_{t}$ on $\Omega_{t-1}$ become easily computationally intractable for practical purposes.

Let $\operatorname{vech}(A)$ denote the half-vectorization operator stacking the elements of a quadratic $(N \times N)$-matrix $A$ from the main diagonal downwards in a $\frac{1}{2} N(N+$ 1) dimensional column vector. Within the so-called vec-representation of the $\operatorname{GARCH}(p, q)$ model $\Sigma_{t}$ is specified as follows:

$$
\operatorname{vech}\left(\Sigma_{t}\right)=c+\sum_{i=1}^{q} \tilde{A}_{i} \operatorname{vech}\left(\varepsilon_{t-i} \varepsilon_{t-i}^{T}\right)+\sum_{i=1}^{p} \tilde{G}_{i} \operatorname{vech}\left(\Sigma_{t-i}\right) .
$$

In (1.2) the matrices $\tilde{A}_{i}$ and $\tilde{G}_{i}$ each contain $(N(N+1) / 2)^{2}$ elements. Deterministic covariance components are collected in $c$, a column vector of dimension $N(N+1) / 2$. We consider in the following the case $p=q=1$ since in applied work the $\operatorname{GARCH}(1,1)$ model has turned out to be particularly useful to describe a wide variety of financial market data (Bollerslev, Engle and Nelson, 1994).

On the one hand the vec-model in (1.2) allows for a very general dynamic structure of the multivariate volatility process. On the other hand this specification suffers from high dimensionality of the relevant parameter space. Note that the estimation of GARCH processes always requires iterative optimization algorithms. Containing a very large number of model parameters the vec-model is often intractable for empirical work. Apart from computational feasibility it may become cumbersome in applied work to restrict the admissible parameter space such that the implied matrices $\Sigma_{t}, t=1, \ldots, T$, are positive definite.

Variants of multivariate GARCH processes can be evaluated with respect to their scope of handling the two issues raised above: 
- Their ability of economizing on the dimension of the parameter space to make computational exercises feasible.

- Their need of additional restrictions on the parameter space to guarantee positive definiteness of the implied conditional covariance matrices.

Prominent proposals reducing the dimensionality of (1.2) are the constant correlation model (Bollerslev, 1990) and the diagonal model (Bollerslev et al., 1988). Specifying diagonal elements of $\Sigma_{t}$ both of these approaches assume the absence of cross equation dynamics, i.e. the only dynamics are

$$
\sigma_{i i, t}=c_{i i}+a_{i} \varepsilon_{i, t-1}^{2}+g_{i} \sigma_{i i, t-1}, i=1, \ldots, N .
$$

To determine off-diagonal elements of $\Sigma_{t}$ Bollerslev (1990) proposes a constant contemporaneous correlation,

$$
\sigma_{i j, t}=\rho_{i j} \sqrt{\sigma_{i i} \sigma_{j j}}, i, j=1, \ldots, N,
$$

whereas Bollerslev et al. (1988) introduce an ARMA-type dynamic structure as in (1.3) for $\sigma_{i j, t}$ as well, i.e.

$$
\sigma_{i j, t}=c_{i j}+a_{i j} \varepsilon_{i, t-1} \varepsilon_{j, t-1}+g_{i j} \sigma_{i j, t-1}, i, j=1, \ldots, N .
$$

For the bivariate case $(N=2)$ with $p=q=1$ the constant correlation model contains only 7 parameters compared to 21 parameters encountered in the full model (1.2). The diagonal model is specified with 9 parameters. The price that both models pay for parsimonity is in ruling out cross equation dynamics as allowed in the general vec-model. Positive definiteness of $\Sigma_{t}$ is easily guaranteed for the constant correlation model $\left(\left|\rho_{i j}\right|<1\right)$, whereas the diagonal model requires more complicated restrictions to provide positive definite covariance matrices.

The so-called BEKK-model (named after Baba, Engle, Kraft and Kroner, 1990) provides a richer dynamic structure compared to both restricted processes mentioned before. Defining $N \times N$ matrices $A_{i k}$ and $G_{i k}$ and an upper triangular matrix $C_{0}$ the BEKK-model reads in a general version as follows:

$$
\Sigma_{t}=C_{0}^{T} C_{0}+\sum_{k=1}^{K} \sum_{i=1}^{q} A_{i k}^{T} \varepsilon_{t-i} \varepsilon_{t-i}^{T} A_{i k}+\sum_{k=1}^{K} \sum_{i=1}^{p} G_{i k}^{T} \Sigma_{t-i} G_{i k} .
$$


If $K=q=p=1$ and $N=2$, the model in (1.6) contains 11 parameters and implies the following dynamic model for typical elements of $\Sigma_{t}$ :

$$
\begin{aligned}
\sigma_{11, t} & =c_{11}+a_{11}^{2} \varepsilon_{1, t-1}^{2}+2 a_{11} a_{21} \varepsilon_{1, t-1} \varepsilon_{2, t-1}+a_{21}^{2} \varepsilon_{2, t-1}^{2} \\
& +g_{11}^{2} \sigma_{11, t-1}+2 g_{11} g_{21} \sigma_{21, t-1}+g_{21}^{2} \sigma_{22, t-1} \\
\sigma_{21, t} & =c_{21}+a_{11} a_{22} \varepsilon_{1, t-1}^{2}+\left(a_{21} a_{12}+a_{11} a_{22}\right) \varepsilon_{1, t-1} \varepsilon_{2, t-1}+a_{21} a_{22} \varepsilon_{2, t-1}^{2} \\
& +g_{11} g_{22} \sigma_{11, t-1}+\left(g_{21} g_{12}+g_{11} g_{22}\right) \sigma_{12, t-1}+g_{21} g_{22} \sigma_{22, t-1}, \\
\sigma_{22, t} & =c_{22}+a_{12}^{2} \varepsilon_{1, t-1}^{2}+2 a_{12} a_{22} \varepsilon_{1, t-1} \varepsilon_{2, t-1}+a_{22}^{2} \varepsilon_{2, t-1}^{2} \\
& +g_{12}^{2} \sigma_{11, t-1}+2 g_{12} g_{22} \sigma_{21, t-1}+g_{22}^{2} \sigma_{22, t-1} .
\end{aligned}
$$

Compared to the diagonal model the BEKK-specification economizes on the number of parameters by restricting the vec-model within and across equations. Since $A_{i k}$ and $G_{i k}$ are not required to be diagonal, the BEKK-model is convenient to allow for cross dynamics of conditional covariances. The parameter $K$ governs to which extent the general representation in (1.2) can be approximated by a BEKK-type model. In the following we implicitly assume $K=1$. Note that in the bivariate case with $K=p=q=1$ the BEKK-model contains 11 parameters. If $K=1$ the matrices $A_{11}$ and $-A_{11}$, imply the same conditional covariances. Thus, for uniqueness of the BEKK-representation $a_{11}>0$ and $g_{11}>0$ is assumed. Note that the right hand side of (1.6) involves only quadratic terms and, hence, given convenient initial conditions, $\Sigma_{t}$ is positive definite under the weak (sufficient) condition that at least one of the matrices $C_{0}$ or $G_{k i}$ has full rank (Engle and Kroner, 1995).

\subsubsection{Estimation of the BEKK-model}

As in the univariate case the parameters of a multivariate GARCH model are estimated by maximum likelihood (ML) optimizing numerically the Gaussian log-likelihood function.

The contribution of a single observation to the log-likelihood of a sample is given as:

$$
\begin{aligned}
l_{t}(.) & =\ln \left(f\left(\varepsilon_{t} \mid \Omega_{t-1}\right)\right) \\
& =-\frac{N}{2} \ln (2 \pi)-\frac{1}{2} \ln \left(\left|\Sigma_{t}\right|\right)-\frac{1}{2} \varepsilon_{t}^{T} \Sigma_{t}^{-1} \varepsilon_{t} .
\end{aligned}
$$

Maximizing the Gaussian log-likelihood $l=\sum_{t=1}^{T} l_{t}($.$) requires nonlinear max-$ imization methods. Involving only first order derivatives the algorithm intro- 
duced by Berndt, Hall, Hall, and Hausman (1974) is easily implemented and particularly useful for the estimation of multivariate GARCH processes.

If the actual error distribution differs from the multivariate normal, maximizing the Gaussian log-likelihood has become popular as Quasi ML (QML) estimation. In the multivariate framework, results for the asymptotic properties of the (Q)ML-estimator have been derived recently. Jeantheau (1998) proves the QML-estimator to be consistent under the main assumption that the considered multivariate process is strictly stationary and ergodic. Further assuming finiteness of moments of $\varepsilon_{t}$ up to order eight, Comte and Lieberman (2000) derive asymptotic normality of the QML-estimator. The asymptotic distribution of the rescaled QML-estimator is analogous to the univariate case and discussed in Bollerslev and Wooldridge (1992).

\subsection{An empirical illustration}

\subsubsection{Data description}

We analyze daily quotes of two European currencies measured against the USD, namely the DEM and the GBP. The sample period is December 31, 1979 to April 1, 1994, covering $T=3720$ observations. Note that a subperiod of our sample has already been investigated by Bollerslev and Engle (1993) discussing common features of volatility processes.

The data is provided in fx.dat. The first column contains DEM/USD and the second GBP/USD. In XploRe a preliminary statistical analysis is easily done by the summarize command. Before inspecting the summary statistics, we load the data and take log differences. The following XploRe code

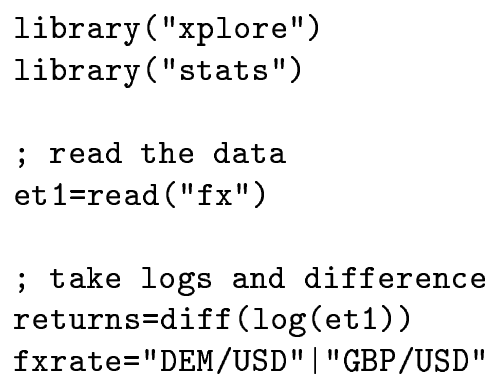


; produce summary statistics

summarize(returns, fxrate)

Q XFGmvol01.xpl

produces the output given below:

\begin{tabular}{|c|c|c|c|c|c|c|}
\hline 2,$]$ & $"$ & Minimum & Maximum & Mean & Median & Std.Error" \\
\hline & & & & & & 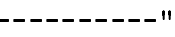 \\
\hline & & $v$ & 0.0 & 4. & 0 & $36 "$ \\
\hline & GBP/USD & -0.046682 & 0.038665 & 0.00011003 & 0 & $0.0069721 "$ \\
\hline
\end{tabular}

Evidently, the empirical means of both processes are very close to zero $(-4.72 \mathrm{e}-$ 06 and 1.10e-04, respectively). Also minimum, maximum and standard errors are of similar size. First differences of the respective log exchange rates are shown in Figure 1.1. As is apparent from Figure 1.1, variations of exchange rate returns exhibit an autoregressive pattern: Large returns in foreign exchange markets are followed by large returns of either sign. This is most obvious in periods of excessive returns. Note that these volatility clusters tend to coincide in both series. It is precisely this observation that justifies a multivariate GARCH specification.

\subsubsection{Estimating bivariate GARCH}

$\{$ th, liks $\}=$ bigarch (theta, et)

estimates a bivariate GARCH model

The quantlet bigarch provides a fast algorithm to estimate the BEKK representation of a bivariate $\operatorname{GARCH}(1,1)$ model. QML-estimation is implemented by means of the BHHH-algorithm which minimizes the negative Gaussian loglikelihood function. The algorithm employs analytical first order derivatives of the log-likelihood function (Lütkepohl, 1996) with respect to the 11-dimensional vector of parameters containing the elements of $C_{0}, A_{11}$ and $G_{11}$ as given in (1.6).

The standard call is

$\{$ coeff, $\operatorname{maxlik}\}=b i g a r c h($ theta, data), 


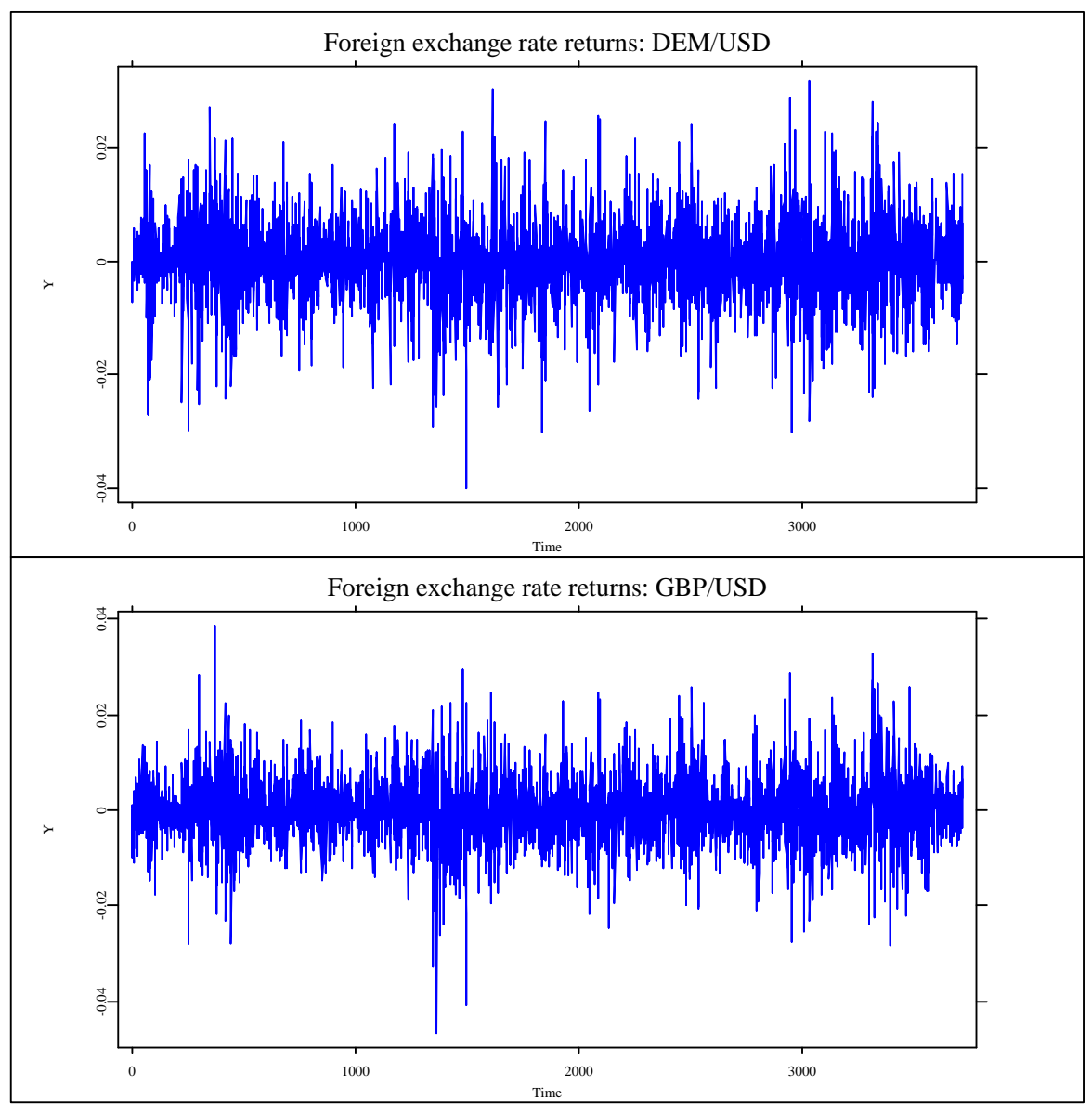

Figure 1.1: Foreign exchange rate data: returns

Q XFGmvol01.xpl

where as input parameters we have initial values theta for the iteration algorithm and the data set, e.g. financial returns, stored in data. The estimation output is the vector coeff containing the elements of the parameter matrices. 
Being a numerical procedure the algorithm requires to determine suitable initial parameters. For the diagonal elements of the matrices $A_{11}$ and $G_{11}$ values around 0.3 and 0.9 appear reasonable, since in univariate $\operatorname{GARCH}(1,1)$ models parameter estimates for $\alpha_{i}$ and $\beta_{i}$ in (1.3) often take values around $0.3^{2}=0.09$ and $0.81=0.9^{2}$. There is no clear guidance how to determine initial values for off diagonal elements of $A_{11}$ or $G_{11}$. Therefore it might be reasonable to try alternative initializations of these parameters. Given an initialization of $A_{11}$ and $G_{11}$ the starting values for the elements in $C_{0}$ are immediately determined by the algorithm assuming the unconditional covariance of $\varepsilon_{t}$ to exist (Engle and Kroner, 1995).

Given our example under investigation the bivariate GARCH estimation could be done by the following pieces of code:

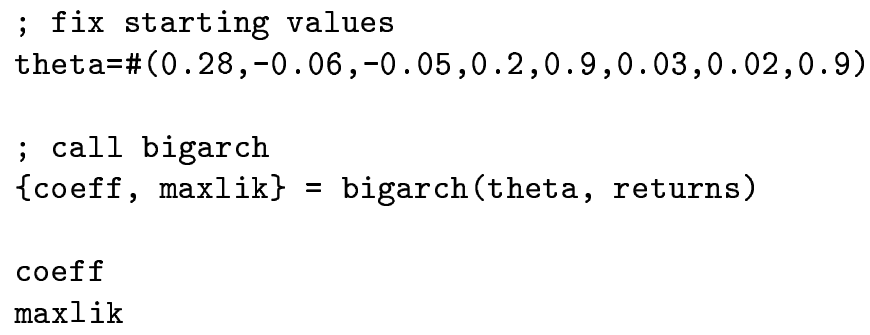

Q XFGmvo102.xpl

As output we obtain

Contents of coeff

$[1] \quad$,

$[2] \quad$,

$[3] \quad$,

$[4] \quad$,

$[5]-$,

$[6]-$,

$\left[\begin{array}{ll}7,] & 0.29344\end{array}\right.$

$[8] \quad$,

$[9] \quad$,

$[10] \quad$,

$[11] \quad$, 
Contents of liks

$[1] \quad-$,

The last number is the obtained minimum of the negative log-likelihood function. The vector coeff given first contains as first three elements the parameters of the upper triangular matrix $C_{0}$, the following four belong to the $\mathrm{ARCH}$ $\left(A_{11}\right)$ and the last four to the GARCH parameters $\left(G_{11}\right)$, i.e. for our model

$$
\Sigma_{t}=C_{0}^{T} C_{0}+A_{11}^{T} \varepsilon_{t-1} \varepsilon_{t-1}^{T} A_{11}+G_{11}^{T} \Sigma_{t-1} G_{11}
$$

stated again for convenience, we find the matrices $C_{0}, A, G$ to be:

$$
\begin{gathered}
C_{0}=10^{-3}\left(\begin{array}{cc}
1.15 & .31 \\
0 & .76
\end{array}\right) \\
A_{11}=\left(\begin{array}{rr}
.282 & -.050 \\
-.057 & .293
\end{array}\right), G_{11}=\left(\begin{array}{ll}
.939 & .028 \\
.025 & .939
\end{array}\right)
\end{gathered}
$$

\subsubsection{Estimating the (co)variance processes}

The (co)variance is obtained by sequentially calculating the difference equation (1.7) where we use the estimator for the unconditional covariance matrix as initial value $\left(\Sigma_{0}=\frac{E^{T} E}{T}\right)$. Here, the $T \times 2$ vector $E$ contains logdifferences of our foreign exchange rate data. Estimating the covariance process is also accomplished in the quantlet XFGmvol02 and additionally provided in sigmaprocess. dat.

Q XFGmvo102.xpI

We display the estimated variance and covariance processes in Figure 1.2. The upper and the lower panel of Figure 1.2 show the variances of the DEM/USD and GBP/USD returns respectively, whereas in the middle panel we see the covariance process. Except for a very short period in the beginning of our sample the covariance is positive and of non-negligible size throughout. This is evidence for cross sectional dependencies in currency markets which we mentioned earlier to motivate multivariate GARCH models. 


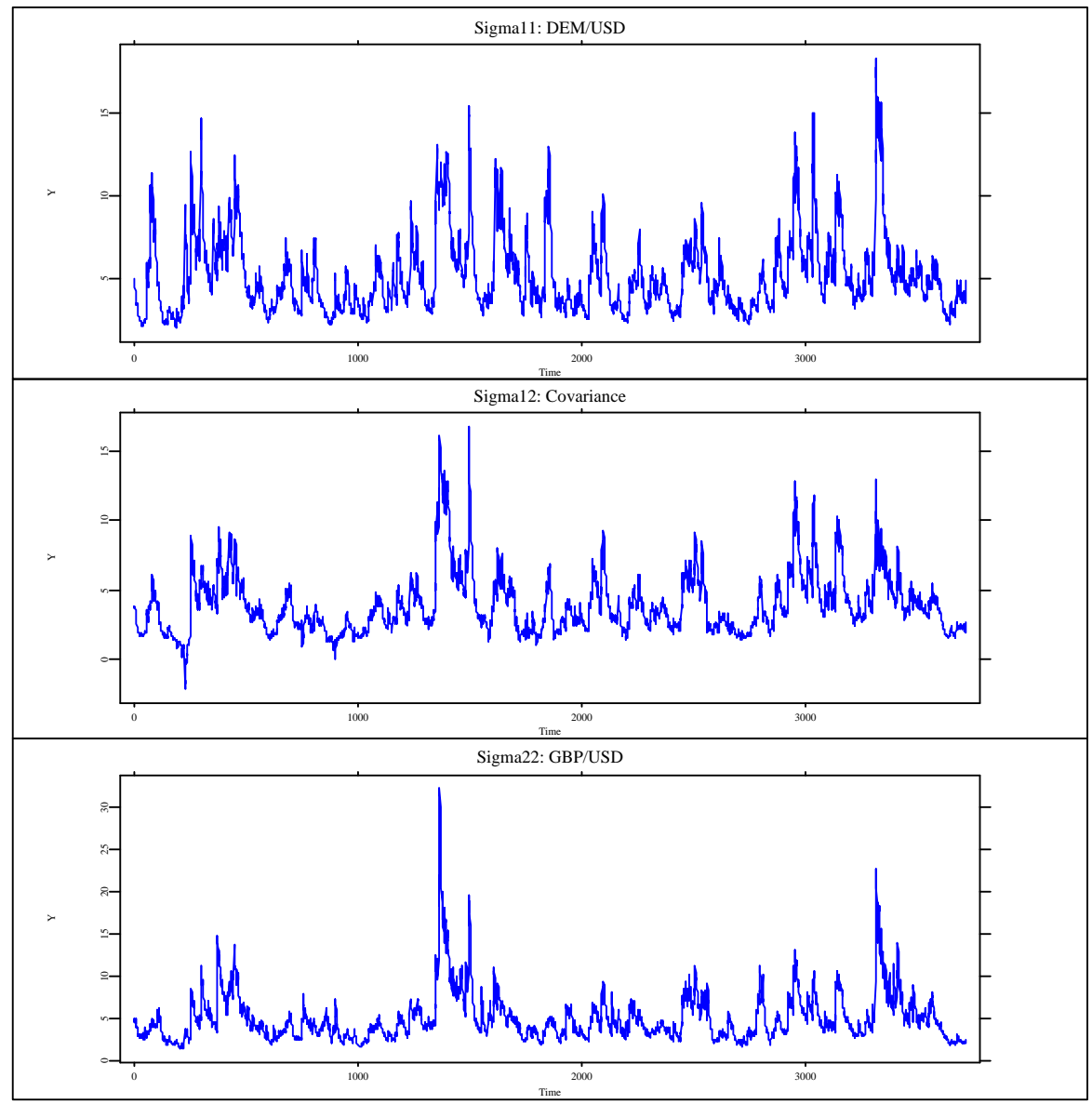

Figure 1.2: Estimated variance and covariance processes $\left(10^{5} \hat{\Sigma_{t}}\right)$

Q. XFGmvo102.xpl

Instead of estimating the realized path of variances as shown above, we could also use the estimated parameters to simulate volatility paths (XFGmvol03). 


\section{Q. XFGmvol03.xpl}

For this at each point in time an observation $\varepsilon_{t}$ is drawn from a multivariate normal distribution with variance $\Sigma_{t}$. Given these observations, $\Sigma_{t}$ is updated according to (1.7). Then, a new residual is drawn with covariance $\Sigma_{t+1}$. We apply this procedure for $T=3000$. The results, displayed in the upper three panels of Figure 1.3, show a similar pattern as the original process given in Figure 1.2. For the lower two panels we generate two variance processes from the same residuals $\xi_{t}$. In this case, however, we set off-diagonal parameters in $A_{11}$ and $G_{11}$ to zero to illustrate how the unrestricted BEKK model incorporates cross equation dynamics. As can be seen, both approaches are convenient to capture volatility clustering. Depending on the particular state of the system, spillover effects operating through conditional covariances, however, have a considerable impact on the magnitude of conditional volatility. 

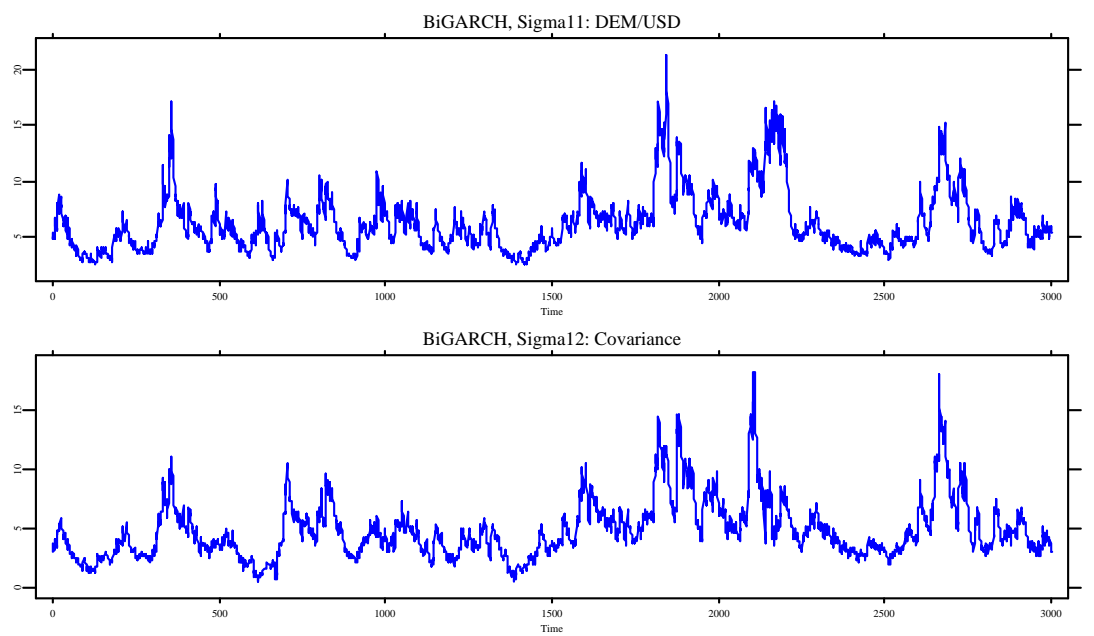

BiGARCH, Sigma22: GBP/USD

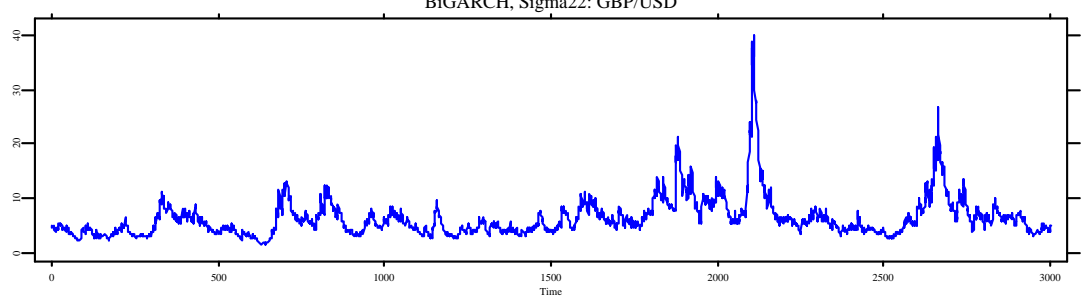

Univariate GARCH, Sigma11: DEM/USD
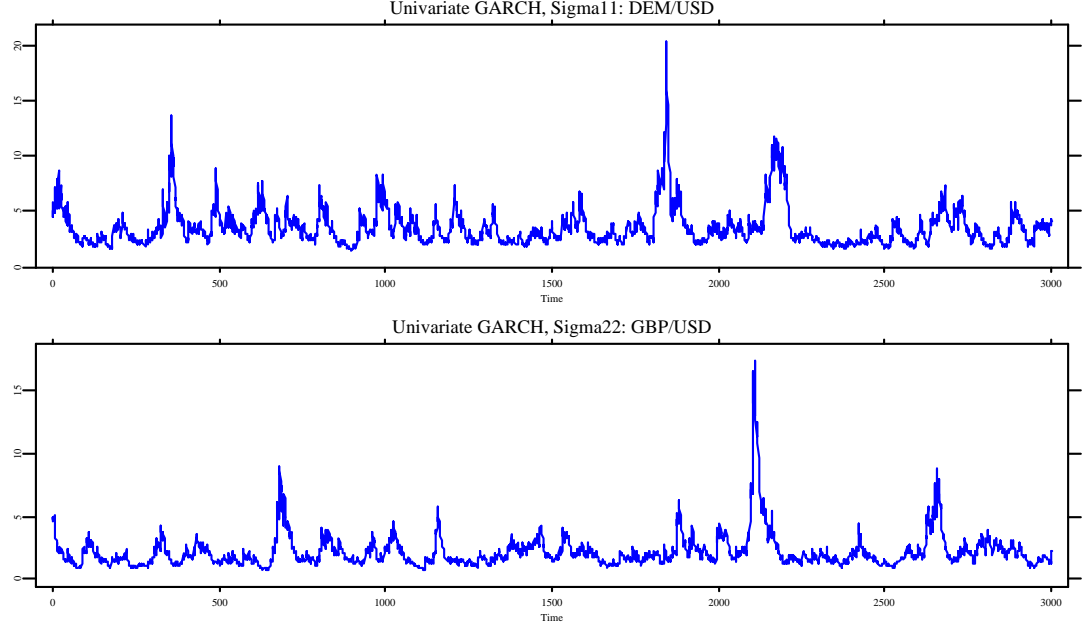

Figure 1.3: A simulated variance and covariance processes, both bivariate and univariate case. $\left(10^{5} \hat{\Sigma}_{t}\right)$

Q XFGmvo103.xpl 


\subsection{An application to option pricing}

The foregoing section illustrated how the GARCH model may be employed effectively to describe empirical price variations of foreign exchange rates. As an economic application of the particular estimated GARCH process one may regard the pricing of derivatives where the investigated foreign exchange rates are the underlyings. In this section we compare foreign exchange rate option prices generated from the bivariate GARCH model estimated before and its univariate competitor. As underlying we consider the DEM/USD-rate.

Pricing options in a GARCH framework has been introduced by Duan (1995) and is extended and applied to major German stocks in Härdle and Hafner (2000) and Hafner and Herwartz (1998). The GARCH option pricing model generalizes the traditional option valuation methodology to the case of conditional heteroskedasticity. Let $P$ denote a subjective probability measure for the price process $S_{t}$. Under some regularity conditions satisfied by the price process, the absence of arbitrage opportunities implies the existence of a probability measure $Q$ equivalent to $P$, such that all discounted prices processes are martingales under $Q$, Harrison and Pliska (1981). Therefore even in the case of conditional heteroskedasticity, the current price of a European call option $C_{t}$ with exercise price $K$ and time to maturity $\tau=T-t$ is given by

$$
C_{t}=(1+r)^{-\tau} E^{Q}\left[\max \left(S_{T}-K, 0\right) \mid \Omega_{t}\right] .
$$

In (1.9) $S_{T}$ denotes the price of the underlying asset in time $T$ and $r$ is the risk-free interest rate. Opposite to the homoskedastic case treated in Black and Scholes (1973) the expectation in (1.9) has been evaluated analytically only for the univariate case in Heston and Nandi (1997). Therefore we determine $C_{t}$ by simulations. The pricing measure $Q$ can be derived from the data generating process specified and estimated for $S_{t}$, the underlying asset. Under specific assumptions, concerning the conditional expectations of returns of $S_{t}$, the measures $Q$ and $P$ coincide. In our case $Q=P$ is implied if both the conditional mean of empirical returns and the risk free rate are assumed to be zero. To focus on the comparison of bivariate and univariate volatility specifications we assume coincidence of $Q$ and $P$. We determine option prices by simulating foreign exchange rates according to the GARCH parameter estimates given in (1.8) and its "univariate" counterpart with off-diagonal elements of $A_{11}$ and $G_{11}$ being zero. We use $R=100000$ replications to evaluate

$$
C_{t}=(1+r)^{-\tau} E^{Q}\left[\max \left(S_{T}-K, 0\right) \mid \Omega_{t}\right] .
$$

Throughout we set $S_{0}=2.00 \mathrm{DEM}, r=0$ and determine option prices for given 


\begin{tabular}{l|lll}
\hline \hline Regime 1 & $\Sigma_{0}=\left(\begin{array}{ll}2.2480 & 1.5748 \\
1.5748 & 1.8946\end{array}\right)$ \\
Regime 2 & $\Sigma_{0}=\left(\begin{array}{cc}6.7449 & -0.5903 \\
-0.5903 & 3.1272\end{array}\right)$ \\
Regime 3 & $\Sigma_{0}=\left(\begin{array}{ll}11.7640 & 14.9770 \\
14.9770 & 27.4520\end{array}\right)$ \\
\hline \hline
\end{tabular}

Table 1.1: Covariance matrices $\left(10^{5} \Sigma_{0}\right)$ among different regimes used as initial values for the Monte Carlo Simulation.

times to maturity, $\tau=30$ days, and varying degrees of moneyness $\left(S_{t} / K\right)$, ranging from 0.85 to 1.15 . We do the simulation for three different regimes of volatility providing the initial state of the dynamic system. These regimes 1 to 3 represent low, medium, and high volatility states, respectively (Table 1.1). All these covariance estimates have been obtained from the estimated variances shown in Figure 1.2 (Regime 1 is the 150th, Regime 2 the 230th, and Regime 3 the 1370 th observation of the covariance process). (To evaluate option prices the reader may choose between these regimes, however, note that the number of replications has been reduced in the quantlet to avoid the computational effort necessary for $R=100000$ replications.).

Q. XFGmvol04.xpl

Initial covariance matrices are displayed in Table 1.1, simulated option prices in Table 1.2. As can readily be seen, prices obtained by the bivariate GARCH are higher throughout. This (positive) absolute price difference reflects an additional risk premium related to the second GARCH process. It has to be positive because options have a one-sided risk profile. It is highest for the at-the-money option $(K=2.00)$, and is decreasing for both in- and out-of-themoney options. This is in line with the Black-Scholes model, where at-themoney options exhibit highest sensitivity to changes in volatility. Among our three regimes, the premium is highest in absolute terms for the high volatility regime 3 (Table 1.2 and Figure 1.4). For the medium volatility regime 2 the price difference is mitigated by the small negative covariance of the initial 


\begin{tabular}{c|ccccccc}
\hline \hline & & & & & & & \\
Moneyness & 0.85 & 0.90 & 0.95 & 1.00 & 1.05 & 1.10 & 1.15 \\
Strikes & 2.3529 & 2.2222 & 2.1053 & 2.0000 & 1.9048 & 1.8182 & 1.7391 \\
& & & & & & & \\
\hline \hline & 0.0000 & $1.93 \mathrm{e}-05$ & 0.0014 & 0.0246 & 0.0979 & 0.1831 & 0.2621 \\
Regime 1 & 0.0000 & $1.46 \mathrm{e}-05$ & 0.0011 & 0.0229 & 0.0974 & 0.1830 & 0.2620 \\
& n.a. & 0.3240 & 0.3207 & 0.0751 & 0.0046 & 0.0007 & 0.0005 \\
\hline & $1.44 \mathrm{e}-05$ & $3.27 \mathrm{e}-04$ & 0.0047 & 0.0331 & 0.1017 & 0.1843 & 0.2630 \\
Regime 2 & $1.29 \mathrm{e}-05$ & $2.98 \mathrm{e}-04$ & 0.0044 & 0.0324 & 0.1014 & 0.1842 & 0.2629 \\
& 0.1150 & 0.0984 & 0.0654 & 0.0217 & 0.0032 & 0.0005 & 0.0003 \\
\hline & 0.0003 & 0.0023 & 0.0135 & 0.0484 & 0.1114 & 0.1883 & 0.2653 \\
Regime 3 & 0.0001 & 0.0011 & 0.0088 & 0.0406 & 0.1062 & 0.1861 & 0.2641 \\
& 1.1943 & 0.9996 & 0.5436 & 0.1907 & 0.0484 & 0.0117 & 0.0045 \\
\hline \hline
\end{tabular}

Table 1.2: Results obtained from the Monte Carlo Simulation: For each regime, the first row displays the prices from the bivariate GARCH, the second row from the univariate GARCH specification. The third row shows relative differences between both. $S_{0}=2.00$.

volatility state. In relative terms (third row for each regime in Table 1.2), the same pattern emerges as for the absolute price difference, however the deviation is highest for out-of-the-money options. This is due to the fact that for in-themoney options the largest fraction of the price is borne by its intrinsic value, i.e. $S_{0}-K$. Since interest rates are zero in our simulations, the prices of in-the-money options almost equal $2.00-K$.

\subsection{Conclusions}

We briefly discussed specification issues relevant for multivariate volatility modeling. The BEKK model is particularly useful for practical purposes, since it allows for cross sectional dynamics and, moreover, positive definiteness of implied volatility matrices holds under rather weak assumptions. In XploRe the quantlet bigarch estimates the BEKK model for two dimensional return processes. Investigating joint volatility dynamics of the DEM/USD and GBP/USD exchange rates, we illustrate the convenience of both the BEKK model and its implementation in XploRe. As one particular issue we show how the (unre- 


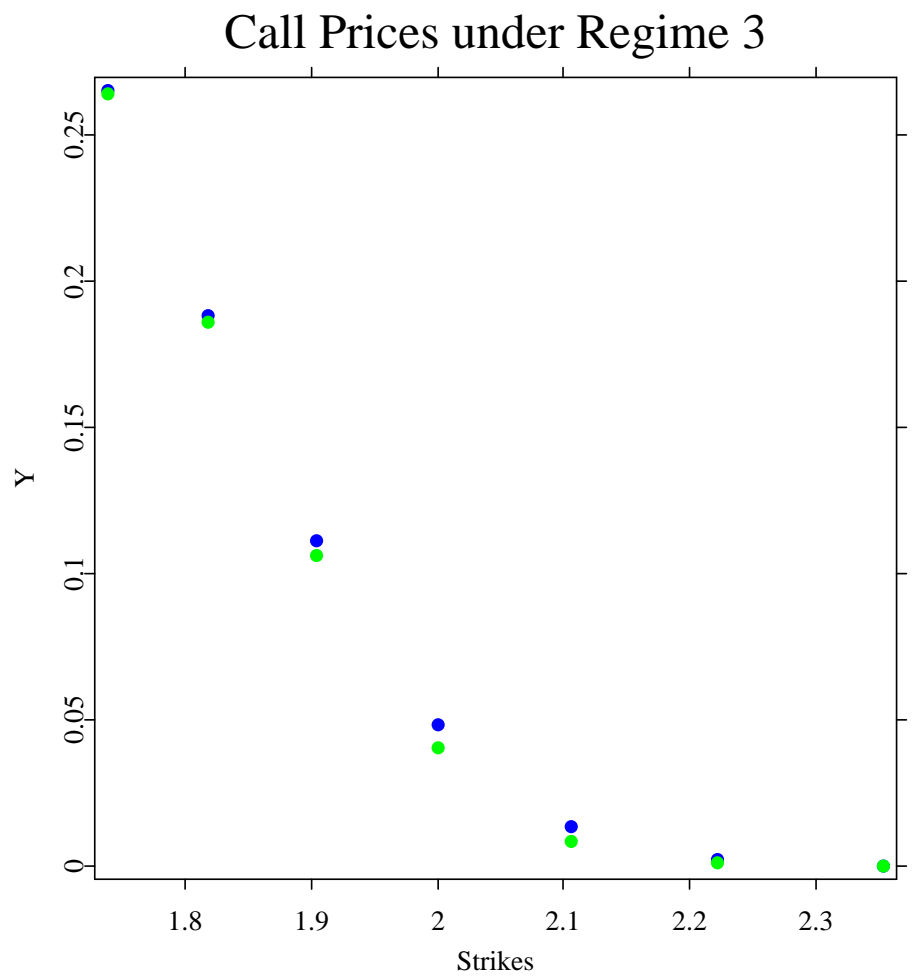

Figure 1.4: Option prices under the high volatility regime - blue are BiGARCH, green are GARCH prices. $R=100000$ replications, $S_{0}=2.00$.

Q XFGmvol04.xpl

stricted) BEKK model performs relative to univariate models when evaluating time dependent second order moments. The former model turns out to capture volatility spillovers which are highly intuitive. Naturally, univariate volatility models, i.e. restricted BEKK-specifications, cannot deliver such insights. Providing an economic application we find that the dimension of the considered volatility state space has an important impact on the level of option prices. 


\section{Bibliography}

Baba, Y., Engle, R.F., Kraft, D.F., and Kroner, K.F. (1990). Multivariate Simultaneous Generalized ARCH, mimeo, Department of Economics, University of California, San Diego.

Berndt, E.K., Hall B.H., Hall, R.E., and Hausman, J.A. (1974). Estimation and Inference in Nonlinear Structural Models, Annals of Economic and Social Measurement 3/4: 653-665.

Black F., and Scholes M. (1973). The Pricing of Options and Corporate Liabilities, Journal of Political Economy 81: 637-659.

Bollerslev, T. (1986). Generalized Autoregressive Conditional Heteroscedasticity, Journal of Econometrics 31: 307-327.

Bollerslev, T. (1990). Modeling the Coherence in Short-Run Nominal Exchange Rates: A Multivariate Generalized ARCH Approach, Review of Economics and Statistics 72: 498-505.

Bollerslev, T. and Engle, R.F. (1993). Common Persistence in Conditional Variances, Econometrica 61: 167-186.

Bollerslev, T., Engle, R.F. and Nelson, D.B. (1994). GARCH Models, in: Engle, R.F., and McFadden, D.L. (eds.) Handbook of Econometrics, Vol. 4, Elsevier, Amsterdam, 2961-3038.

Bollerslev, T., Engle, R.F. and Wooldridge, J.M. (1988). A Capital Asset Pricing Model with Time-Varying Covariances, Journal of Political Economy 96: $116-131$.

Bollerslev, T. and Wooldridge, J.M. (1992). Quasi-Maximum Likelihood Estimation and Inference in Dynamic Models with Time-Varying Covariances, Econometric Reviews, 11: 143-172. 
Cecchetti, S.G., Cumby, R.E. and Figlewski, S. (1988). Estimation of the Optimal Futures Hedge, Review of Economics and Statistics 70: 623-630.

Comte, F. and Lieberman, O. (2000). Asymptotic Theory for Multivariate GARCH Processes, Manuscript, Universities Paris 6 and Paris 7.

Duan J.C. (1995). The GARCH Option Pricing Model, Mathematical Finance 5: $13-32$.

Engle, R.F. (1982). Autoregressive Conditional Heteroscedasticity with Estimates of the Variance of UK Inflation. Econometrica 50: 987-1008.

Engle, R.F., Ito, T. and Lin, W.L. (1990). Meteor Showers or Heat Waves? Heteroskedastic Intra-Daily Volatility in the Foreign Exchange Market, Econometrica 58: 525-542.

Engle, R.F. and Kroner, K.F. (1995). Multivariate Simultaneous Generalized ARCH, Econometric Theory 11: 122-150.

Härdle, W. and Hafner, C. (2000). Discrete Time Option Pricing with Flexible Volatility Estimation, Finance and Stochastics 4: 189-207.

Hafner, C.M. and Herwartz, H. (1998). Structural Analysis of Portfolio Risk using Beta Impulse Response Functions, Statistica Neerlandica 52: 336355 .

Hafner C.M. and Herwartz, H. (2001). Option Pricing under Linear Autoregressive Dynamics, Heteroskedasticity, and Conditional Leptokurtosis, Journal of Empirical Finance 8: 1-34.

Hamao, Y., Masulis, R.W. and Ng, V.K. (1990). Correlations in Price Changes and Volatility across International Stock Markets, Review of Financial Studies 3: 281-307.

Harrison, J. M. and Pliska, S. (1981). Martingales and Stochastic Integrals in the Theory of Continuous Trading, Stochastic Processes and their Applications 11: 215-260.

Heston, S.L. and Nandi, S. (1997). A CLosed-Form GARCH Option Pricing Model, Working Paper 97-9, Federal reserve Bank of Atlanta.

Jeantheau, T. (1998). Strong Consistency of Estimators for Multivariate ARCH Models, Econometric Theory 14: 70-86.

Lütkepohl, H. (1996). Handbook of Matrices, Wiley, Chichester. 
Nelson, D.B. (1991). Conditional Heteroskedasticity in Asset Returns: A New Approach, Econometrica 59: 347-370. 